\title{
Characterization of Outpatients With Systolic Dysfunction in a Japanese Community by Total Enumeration Sado Heart Failure Study
}

\author{
Yuji Okura, MD; Yukiko Ohno, MD; Keisuke Suzuki, MD*; Koji Taneda, MD*; \\ Mahmoud M Ramadan, MD; Wataru Mitsuma, MD; Komei Tanaka, MD; \\ Takeshi Kashimura, MD; Masahiro Ito, MD; Osamu Ishizuka, MD**; \\ Kiminori Kato, MD; Haruo Hanawa, MD; Yasuyuki Honda, MD'; \\ Makoto Kodama, MD; Yoshifusa Aizawa, MD
}

\begin{abstract}
Background The prevalence of congestive heart failure (CHF) is increasing with the aging of the community. Management of patients with systolic dysfunction (SD) is important for prevention of CHF, but there is little information regarding the burden of SD on Japanese communities.

Methods and Results In order to delineate the epidemiological and clinical characteristics of SD patients, the medical records of patients from Sado Island were collected and summarized in 2003. From the 5 years prior to 2003, data for 497 patients were extracted. The mortality rate was significantly higher compared with the general population; and the total number of survivors had decreased to 410 by 2003. The proportion of SD patients in the general population increased sharply after the age of 65 years in males and 70 years in females, reaching $3.3 \%$ and $1.7 \%$ for men and women, respectively, in their $80 \mathrm{~s}$. In $49 \%$ of the patients, the Charlson comorbidity index was $\geq 2$, whereas $24 \%$ of females led a solitary life.

Conclusions The total count of outpatients with SD is progressively increasing with age. These patients have multiple comorbidities, making the outcome of SD a poor one. The gender difference in disease characteristics and living conditions should be taken into consideration when establishing preventive strategies for CHF in Japanese communities. (Circ J 2007; 71: 1004-1012)
\end{abstract}

Key Words: Charlson comorbidity index; Heart failure; Systolic dysfunction

$\mathbf{I}$ n developed countries, the number of patients with heart failure (HF) is increasing progressively with the aging of the population, and the "New Epidemic" which Braunwald predicted at the end of the 20th century has now come true! Population-based studies in the United States and Europe have indicated that a large number of individuals with left ventricular (LV) systolic dysfunction (SD) are "hidden" behind those with symptomatic chronic $\mathrm{HF}^{2-4}$ Therefore, guidelines for the management of HF emphasize the early detection and treatment of patients with $\mathrm{SD}$ as an important step in the strategy to reduce this burden in the future 5,6 Management of SD may also be important for reducing the future burden in Asian communities, where aging of the populating is rapidly advancing?

Japan has a universal health insurance system for treatment of diseases and local governments provide financial support for medical check-ups aimed at early detection of diseases. Because of good accessibility to medical services,

(Received December 25, 2006; revised manuscript received March 22, 2007; accepted April 11, 2007)

Division of Cardiology, First Department of Internal Medicine, Niigata University Graduate School of Medical and Dental Sciences, Niigata, *Sado General Hospital, **Ryotsu Hospital and Honda Clinic, Sado, Japan

Mailing address: Yuji Okura, MD, Division of Cardiology, Niigata University Graduate School of Medical and Dental Sciences, 1-754 Asahimachi, Niigata 951-8510, Japan. E-mail: okuray@med.niigata-u. ac.jp a considerable percentage of residents have been screened for heart disease, so much information has accumulated in the local medical centers. Not a few patients are presumed to be under treatment for SD; however, scarce information is available. Therefore, in order to delineate the epidemiological and clinical characteristics of patients with SD in January 2003, we carried out a complete enumeration of the patients in Sado City (an island city in Japan) via cooperation with the local core hospitals and medical societies.

\section{Methods}

\section{Study Design and Participants}

The Niigata-Sado Heart Failure Study was a complete enumeration survey for outpatients with SD at hospitals and clinics in 2 cities in Japan: Niigata and Sado (Fig 1). Sado City is an island with a surface area of $855 \mathrm{~km}^{2}$ (about 1.4-fold the size of Tokyo metropolis), having a total population of 70,000 (almost all Japanese). This study was carried out at 4 core hospitals with hospitalization capacity, in collaboration with the local medical society. SD was defined as LV ejection fraction (LVEF) $\leq 50 \%$. Within a total period of 6 years (extending from January $1^{\text {st }} 1997$ to December $31^{\text {st }} 2002$ ), data for patients with SD were extracted from the records of approximately 7,982 consecutive individuals who had their echocardiographic (Echo) examinations performed at 3 of the core hospitals in Sado City. 


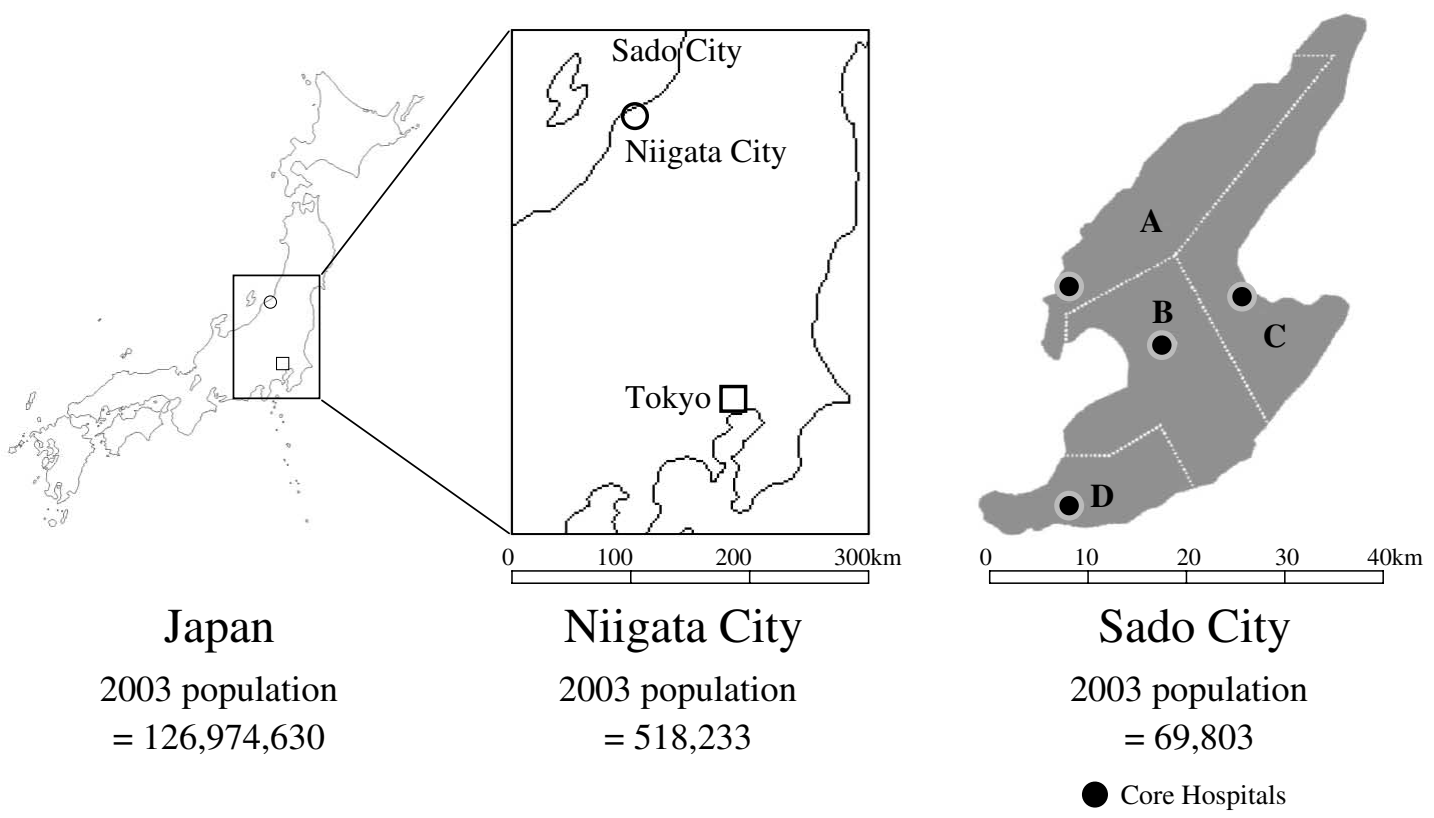

Fig 1. Map of the location and population of Niigata City and Sado Island in Japan on January 1st 2003; with details of the study area and its administrative divisions.

Niigata City is the nearest port to Sado City and has a population of 518,233 persons (Fig 1). Similarly, we examined all records of patients who underwent Echo examination in 14 hospitals in Niigata City to extract the total number of SD patients there.

\section{Determination of Recruitment Period}

The extraction of SD patients was begun on January $1^{\text {st }}$ for 6 consecutive years (such as 2002, 2001, 2000, 1999, 1998, and 1997), and continued until December 31 2002. Thus, SD patients were extracted from the Echo logs by tentatively setting the recruitment periods at $1,2,3,4,5$, and 6 years. We noticed that the number of extracted patients increased with the extension of the recruitment period. However, the earlier the patients were identified, the more information was lost before 2003 because of death or missing data. Therefore, we compared the total counts of recruited patients in these 6 intervals to find the optimal period for identifying the proportion of SD patients in the general population in 2003. In case the same patient was extracted twice or more during the recruitment period, only data from the first extraction was considered.

\section{Review of Cases}

All medical records of the extracted patients were reviewed, and their clinical data were collected and summarized according to a predetermined protocol. If a patient consulted 2 or more institutions during the recruitment period, all the records at these institutions were reviewed.

\section{Extraction of Patients' Data}

The available clinical records were reviewed with regard to demography, medical history, SD etiology, associated comorbidities or complications, medical therapies, laboratory investigations, ECG and Echo examinations. For the patients who survived until 2003, the information was collected up to January $1^{\text {st }} 2003$. Present or past history of congestive HF (CHF) was confirmed using the Framingham criteria8

\section{Determination of SD Etiology}

Three cardiologists (Y Okura, K Suzuki, K Taneda) collaboratively consulted and diagnosed the etiology of SD. Ischemic heart disease (IHD) was considered as an etiology if the patient had 1 of the following: (1) documented history of myocardial infarction (MI), angina or prior coronary revascularization; (2) pathologic Q waves on ECG; or (3) $>75 \%$ stenosis in 1 or more coronary arteries on coronary angiograms. Dilated cardiomyopathy (DCM) was diagnosed by the presence of global LV dilatation with impaired systolic function occurring in the absence of known cardiac or systemic causes. Valvular heart disease (VHD) was considered as an etiology of SD if mitral or aortic valve involvement was documented by Echo in the absence of IHD or DCM. Hypertensive heart disease was considered present if there was a history of hypertension and LV hypertrophy confirmed by Echo.

\section{Confirmation of Survival}

The survival of SD patients up to or beyond January $1^{\text {st }}$ 2003 was confirmed by medical records. For those lacking records up to that date, direct phone calls to the family or attending physician were undertaken.

\section{Determination of the Proportion of SD Patients in the General Population}

This was done using the following equation:

$$
\frac{\text { No. of SD survivors up to January } 1^{\text {st }} 2003}{\text { Estimated population of Sado City on January } 1^{\text {st }} 2003} \times 100
$$

The numerator represents the Echo-documented SD patients extracted within the 5-year period after excluding those for whom survival status was impossible to determine. The denominator estimate was obtained from the Niigata Prefectural Department of Policy and Planning, Niigata, 


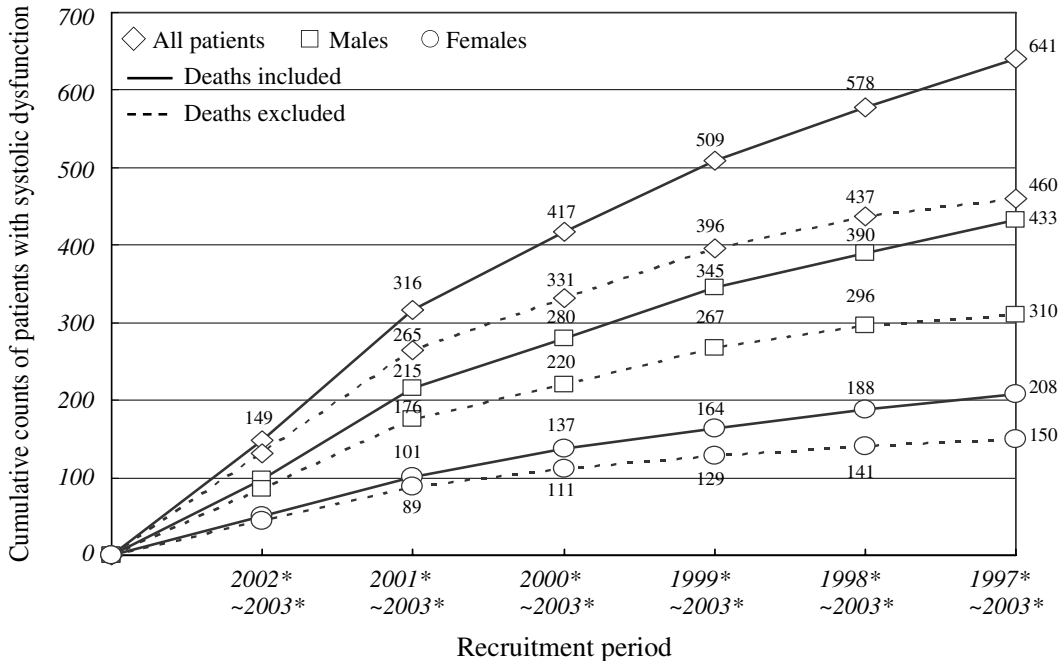

Patients* with systolic dysfunction $(\mathrm{EF} \leq 50 \%)$

recruited from 1998 to $2002(\mathrm{n}=578)$

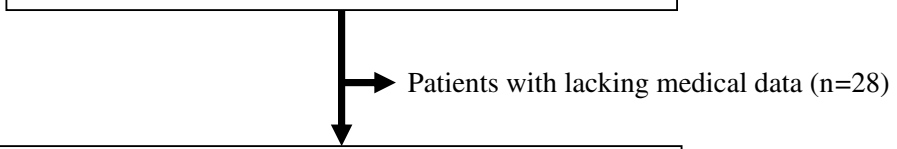

Patients with complete medical data $(\mathrm{n}=550)$

In-hospital deaths $(\mathrm{n}=53)$

Outpatients followed-up $(n=497)$

Deaths before January $1^{\text {st }} 2003(n=87)$

Survivors until January $1^{\text {st }} 2003$ eligible for inclusion in our study $(n=410)$
Fig 2. Cumulative frequency plot showing the yearly-partitioned recruitment period and the corresponding numbers of patients with systolic dysfunction extracted from the echocardiography files up to January $1^{\text {st }} 2003$. The increase in the number of patients was negligible when the recruitment period exceeded 5 years (dotted line). The number of patients missed because of a lack of medical records also increased with prolongation of the recruitment period (not shown). *January $1^{\text {st }}$ of each year.
Fig 3. Flow chart of Sado Heart Failure study, showing the process of extraction and follow-up of patients with systolic dysfunction. When the starting date of the recruitment period was set at 5 years (from January $1^{\text {st }} 1998$ until December $31^{\text {st }} 2002$ ), 578 patients were extracted. Of them, 168 patients were excluded because of a lack of medical records, in-hospital (pre-discharge) mortality, or death during follow-up. Thus, 410 patients finally survived until January $1^{\text {st }} 2003$ and were included in the analysis. *Residents of Sado City with expected age range $45-84$ years at January $1^{\text {st }} 2003$.
Japan? The obtained proportion was compared after stratification by gender and 5-year age intervals.

\section{Survival Analysis}

Survival curves of the general population of Sado City were prepared for both genders on the basis of the Niigata Prefecture Life Table (2000) using actuarial analysis! ${ }^{10,11}$ The latter table was made according to the National Life Table, which is based on the results of the Japan Population Census (2000). Similarly; survival curves of patients with LVEF $\leq 40 \%$ and those with LVEF $41-50 \%$ at the time of diagnosis were prepared, stratified by gender, and compared with the general population curves by log-rank test. Each of these curves was drawn by linking the points representing the average survival rate of each 5-year age group.

In addition, we evaluated the effects of associated comorbidities (included or not in the Charlson comorbidity index $(\mathrm{CCI}))^{12,13}$ on patient's survival. Although CCI is a survival index, it has also been validated to correlate with physical disabilities and the re-admission rate in patients with chronic HF!3,14

\section{Statistical Analysis}

Data analyses were performed using SPSS for Windows Version 13 (SPSS Inc, Chicago, IL, USA). Results for continuous normal data were expressed as mean \pm standard deviation. Comparison of means of continuous normal variables across a grouping variable with 2 was undertaken using Student's t-test. Comparison of proportions was made by $X^{2}$ test with Yates's correction. A 2-sided significance level of 0.05 was used for all analyses.

\section{Ethical Considerations}

The study protocol was reviewed and accepted by the local ethical review board of each medical institution participating in this study, including the Niigata University Graduate School of Medical and Dental Sciences, and written consent was given by all patients.

\section{Results}

\section{Evaluating the Cumulative Counts of SD Patients}

The cumulative counts of SD patients recruited during the periods of varying length (ie, from 1 to 6 years) are shown in Fig 2. In order to determine the appropriate re- 

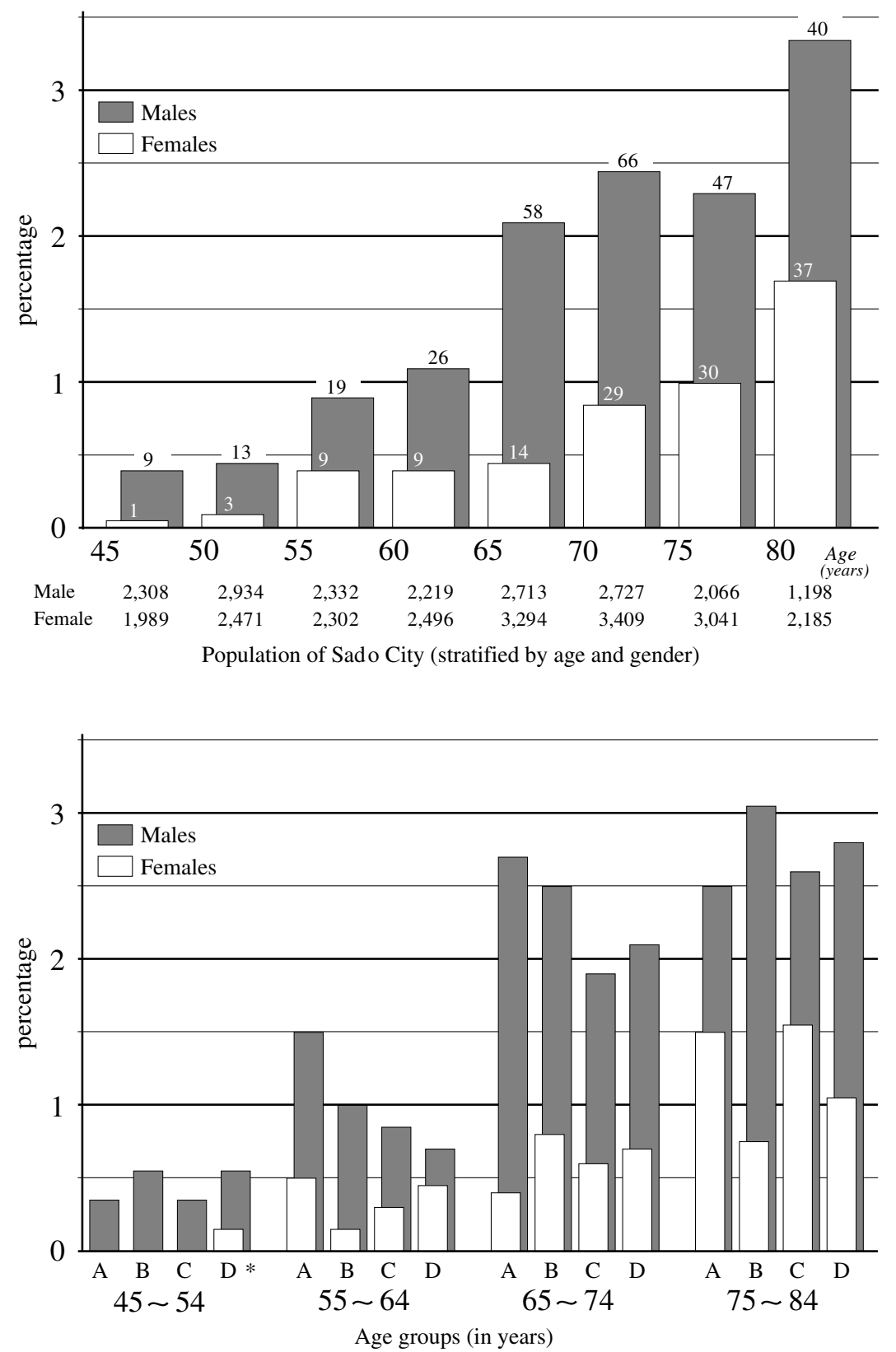

Fig 4. Bar graphs showing the proportion of patients with systolic dysfunction (SD) in the general population on January $1^{\text {st }} 2003$. The number shown at the upper end of each bar represents the SD patients' count, while the numbers below each age range along the horizontal axis represent the male and female populations for that range.
Fig 5. Bar graphs showing the regional proportions of patients with systolic dysfunction (SD) in the general population in Sado City on January $1^{\text {st }} 2003$. there is no significant difference regarding these proportions among the 4 regions. *The letters A to D represent the 4 divisions of Sado City (Island). cruitment period for counting surviving patients in 2003, we evaluated the relationship between recruitment period and the cumulative counts of SD patients. Because some of the recruited patients died before 2003, the number of SD patients who were alive was somewhat fewer than those who were recruited. As shown in Fig 2, solid lines connect the total counts of recruited patients, including those who died before 2003 (Deaths included) and the dotted lines connect the counts of recruited patients who survived until 2003 (Deaths excluded). Considering the yearly intervals as a continuum, a significant positive trend was found for the cumulative counts of SD patients in all patients and in both genders (Deaths included). Nevertheless, such an estimate showed a weak trend relative to the increment of the recruitment period when considering only the cumulative numbers of survivors; that is, the patients' cumulative count curve became nearly flat on moving from 2002-2003 to 1997-2003 (Deaths excluded).

\section{Follow-up of Extracted Patients}

A total of 578 patients were extracted within the 5-year recruitment period (from January $1^{\text {st }} 1998$ till December $31^{\text {st }}$ 2002) (Fig 2) and of them 28 (5\%) were excluded because of either the absence of medical records or inability to confirm survival (Fig 3). Moreover, 53 in-hospital deaths (before discharge) were excluded, as well as 87 deaths that were ascertained to have taken place before January $1^{\text {st }}$ 2003 on careful follow-up. Thus, 410 patients were confirmed to have survived up to that date and were eventually included in our study. We found 18 Sado residents with SD in the Echo logs of the Niigata hospitals, but they had already been diagnosed as SD in Sado.

\section{Proportion of SD Patients in the General Population in Relation to Age, Gender, and Region}

The proportion of SD patients in the general population sector aged $45-84$ years was $1.5 \%$ for males and $0.6 \%$ for 


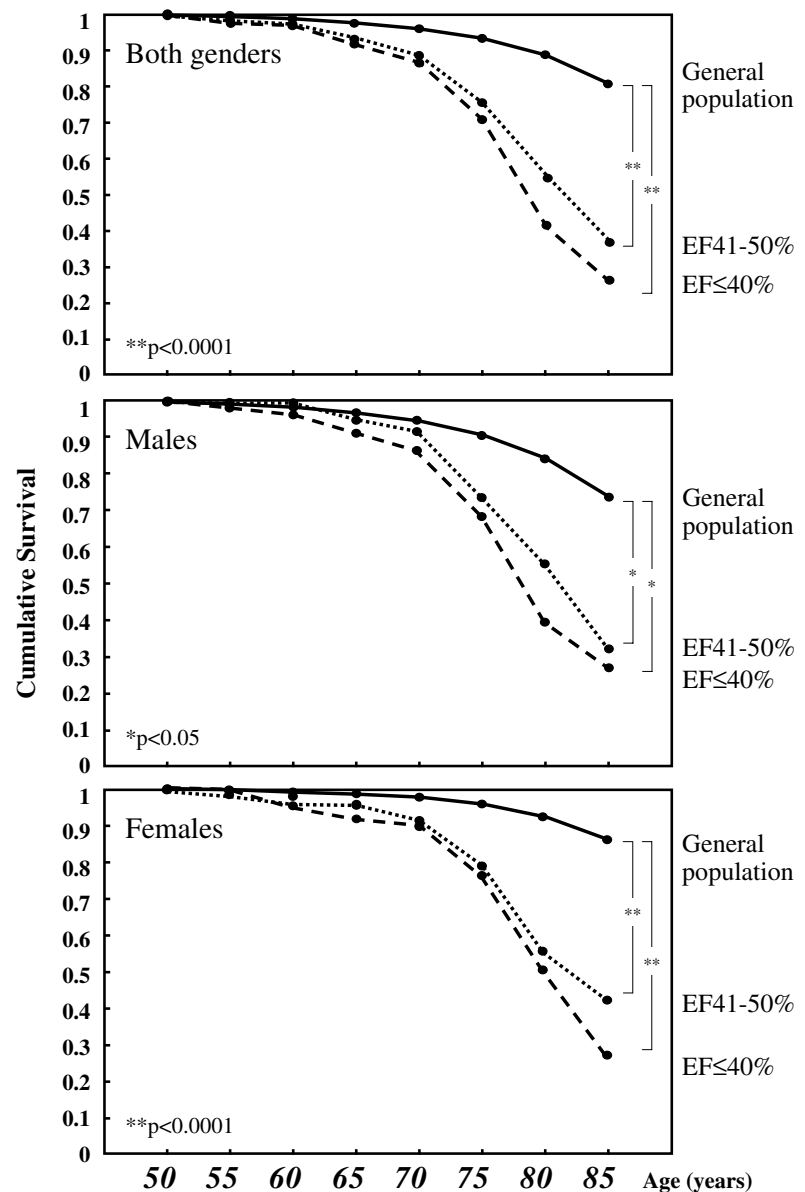

Fig 6. Comparative analysis of survival between patients with systolic dysfunction (SD) and the general population. Survival curves of the general population (on the basis of the Niigata Prefecture Life Table [2000]), and those of patients with mild (left ventricular ejection fraction (LVEF) 41-50\%) and severe (LVEF $\leq 40 \%$ ) SD were prepared using actuarial methods; and comparisons were made by log-rank test. Compared with the general population, the survival rate was significantly lower in both the mild and severe SD patient groups. On the other hand, this rate did not significantly differ between the 2 groups.

females (Fig 4). This proportion increased sharply after the age of 65 years in males and 70 years in females; reaching $3.3 \%$ in males and $1.7 \%$ in females in their $80 \mathrm{~s}$. The proportion in the 4 zones of Sado Island (labeled A-D in Fig 1) was compared after stratification by gender and age decades (Fig 5) and no significant difference in that proportion was found in either gender within any age group among the 4 areas.
Comparison of Patients' Survival Rates Relative

to the General Population

We followed 497 outpatients with SD from diagnosis. The duration of follow-up was $4.5 \pm 2.3$ years. Fig 6 shows survival curves of the general population (based on the life table), compared with those of SD patients, before and after stratification by gender. In comparison with the general population, the cumulative survival was significantly lower in patients with mild SD (LVEF 41-50\%) as well as in those with severe SD (LVEF $\leq 40 \%)$. However, this rate was not significantly different between the 2 groups.

\section{Characteristics of SD Patients}

Demographic Characteristics We included 410 patients with SD whose survivalp until January $1^{\text {st }} 2003$ was correctly confirmed. Approximately two-thirds of them $(68 \%)$ were males, who were significantly younger than the females $(70.2 \pm 8.2$ vs $73.9 \pm 8.6$ years; $\mathrm{p}<0.01)$.

Primary Etiologies of SD Two hundred and three patients $(50 \%)$ underwent diagnostic coronary angiography. As shown in Table 1, IHD was the most frequent etiology of $\mathrm{SD}$ in all cases (34\%) and in males (41\%), whereas VHD was the most prevalent in females (24\%). Hypertensive heart disease was the second important cause of in all cases $(21 \%)$. The primary disease could not be determined in $10 \%$ of cases, particularlyin those with mild SD.

Associated Comorbidities Adequately documented present or past histories of comorbidities are shown for both genders in Table 2. Hypertension was the most prevalent comorbidity found in all cases $(\approx 41 \%)$ and males (42\%). CHF was significantly associated with SD and was the second most prevalent comorbidity in all cases (29\%) and the first one in females $(42 \%)$. MI occurred more often in males $(32 \%)$ than did CHF $(23 \%)$ which came next in importance. Notably; cerebral stroke was markedly prevalent in SD patients (20\% of all cases), especially males $(23 \%$ compared with $14 \%$ in females); as well as psychiatric disorders, such as depression and dementia, which were found in $13 \%$ of patients.

Level of Daily Activities and Lifestyle As can be ascertained from Table 2, the majority of patients (92\%) were independently mobile and wheel-chair-dependents constituted the minority $(3 \%)$. Notably, women tended to lead a more solitary life than men who tended to live in families.

$C C I$ In this index, a score of 1 point is given for each of the following conditions: CHF, MI, and diabetes. Thus, nearly one-quarter $(24 \%)$ of patients were free of comorbidities (a score of 0 ), and similar proportions (27\% and $25 \%$ ) had scores of 1 and 2, respectively. Half of the patients had a CCI score $\geq 2$, which was considered to indicate a poor prognosis. Approximately $12 \%$ of patients had a score $\geq 4$, indicating a low possibility of survival 14

Table 1 Primary Etiologies of SD in the Study Patients

\begin{tabular}{lccc}
\hline \hline Primary etiology of SD & Male $(n=278)$ & Female $(n=132)$ & $p$ value \\
\hline Ischemic heart disease & $113(40.6 \%)$ & $26(19.7 \%)$ & $<0.01$ \\
Hypertensive heart disease & $60(21.6 \%)$ & $27(20.5 \%)$ & $N S$ \\
Valvular heart disease & $26(9.4 \%)$ & $32(24.2 \%)$ & $<0.01$ \\
Dilated cardiomyopathy & $39(14 \%)$ & $14(10.6 \%)$ & $N S$ \\
Chronic renal failure & $8(2.9 \%)$ & $4(3.0 \%)$ & $N S$ \\
Tachyarrhythmias & $6(2.2 \%)$ & $8(6.1 \%)$ & $<0.01$ \\
Congenital cardiac malformations & $5(1.8 \%)$ & $0(0 \%)$ & $N S$ \\
\hline
\end{tabular}

SD, systolic dysfunction. 
Table 2 Clinical and Other Characteristics of Surviving Patients With SD*

\begin{tabular}{|c|c|c|c|}
\hline & Male $(n=278)$ & Female $(n=132)$ & $p$ value \\
\hline \multicolumn{4}{|l|}{ Associated comorbidities and their management } \\
\hline \multicolumn{4}{|l|}{ Cardiovascular risk factors/chronic medical illnesses } \\
\hline Age (years) & $70.2 \pm 8.9$ & $73.9 \pm 8.6$ & $<0.01$ \\
\hline Hypertension & $117(42.1 \%)$ & $49(37.1 \%)$ & NS \\
\hline Diabetes mellitus & $82(29.5 \%)$ & $18(13.6 \%)$ & $<0.01$ \\
\hline Dyslipidemia & $54(19.4 \%)$ & $25(18.9 \%)$ & NS \\
\hline \multicolumn{4}{|l|}{ Body mass index } \\
\hline$\geq 25$ to $<30 \mathrm{~kg} / \mathrm{m}^{2}$ (overweight) & $47(16.9 \%)$ & $13(9.6 \%)$ & $<0.05$ \\
\hline$\geq 30 \mathrm{~kg} / \mathrm{m}^{2}$ (obesity) & $5(1.7 \%)$ & $4(2.7 \%)$ & NS \\
\hline \multicolumn{4}{|l|}{ Cardiac disorders } \\
\hline CHF hospital admission & $65(23.4 \%)$ & $55(41.7 \%)$ & $<0.01$ \\
\hline Angina pectoris & $34(12.2 \%)$ & $4(3 \%)$ & $<0.01$ \\
\hline Myocardial infarction & $89(32.0 \%)$ & $24(18.2 \%)$ & $<0.01$ \\
\hline \multicolumn{4}{|l|}{ Arrhythmias } \\
\hline \multicolumn{4}{|l|}{ Atrial fibrillation } \\
\hline Paroxysmal & $31(11.2 \%)$ & $9(6.8 \%)$ & NS \\
\hline Chronic & $55(19.8 \%)$ & $33(25.0 \%)$ & NS \\
\hline Sustained ventricular tachyarrhythmia & $5(1.8 \%)$ & $2(1.5 \%)$ & NS \\
\hline Permanent cardiac pacemaker & $8(2.9 \%)$ & $0(0 \%)$ & $<0.05$ \\
\hline Intraventricular cardiovertor defibrillator & $0(0 \%)$ & $1(0.8 \%)$ & NS \\
\hline \multicolumn{4}{|l|}{ Cardiac surgery/intervention } \\
\hline Cardiac valve replacement & $12(4.3 \%)$ & $10(7.6 \%)$ & NS \\
\hline Diagnostic coronary angiography & $151(54.3 \%)$ & $52(39.4 \%)$ & NS \\
\hline Percutaneous coronary intervention & $45(16.2 \%)$ & $12(9.1 \%)$ & NS \\
\hline Coronary artery bypass graft operation & $23(8.3 \%)$ & $3(2.3 \%)$ & $<0.05$ \\
\hline \multicolumn{4}{|l|}{ 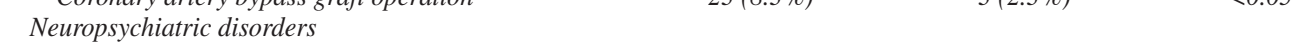 } \\
\hline Stroke & $63(22.7 \%)$ & $18(13.6 \%)$ & $<0.05$ \\
\hline Psychiatric illness & $31(11.2 \%)$ & $22(16.7 \%)$ & NS \\
\hline \multicolumn{4}{|l|}{ Chronic kidney disease } \\
\hline$C R I$ (serum creatinine $\geq 2 \mathrm{mg} / \mathrm{dl}$ ) & $10(3.6 \%)$ & $6(4.5 \%)$ & NS \\
\hline CRF (on regular hemodialysis) & $16(5.8 \%)$ & $4(3.0 \%)$ & NS \\
\hline \multicolumn{4}{|l|}{ Pulmonary disorders } \\
\hline Bronchial asthma & $16(5.8 \%)$ & $1(0.8 \%)$ & $<0.05$ \\
\hline Chronic obstructive pulmonary disease & $18(6.5 \%)$ & $2(1.5 \%)$ & $<0.05$ \\
\hline Home oxygen therapy & $6(2.2 \%)$ & $1(0.8 \%)$ & NS \\
\hline \multicolumn{4}{|l|}{ Malignancy } \\
\hline Nonmetastatic & $23(8.3 \%)$ & $14(10.6 \%)$ & \\
\hline Advanced & $10(3.5 \%)$ & $3(2.3 \%)$ & NS \\
\hline Level of daily activities and lifestyle & & & \\
\hline Independently mobile & $263(94.6 \%)$ & $115(87.2 \%)$ & \\
\hline Wheel chair-dependent & $6(2.0 \%)$ & $6(4.3 \%)$ & $<0.05$ \\
\hline Bedridden & $9(3.4 \%)$ & $11(8.5 \%)$ & \\
\hline Solitary life & $35(12.7 \%)$ & $31(23.6 \%)$ & $<0.01$ \\
\hline Educational background & & & \\
\hline$\leq 12$ years & $241(86.7 \%)$ & $119(90.3 \%)$ & \\
\hline$>12$ years & $37(13.3 \%)$ & $13(9.7 \%)$ & NS \\
\hline Medications & & & \\
\hline$A C E I S$ & $98(35.3 \%)$ & $42(31.8 \%)$ & NS \\
\hline Angiotensin-II receptor blockers & $32(11.5 \%)$ & $6(4.5 \%)$ & $<0.05$ \\
\hline$\beta$-adrenergic receptor blockers & $51(18.3 \%)$ & $12(9.1 \%)$ & $<0.05$ \\
\hline Calcium-channel blockers & $92(33.1 \%)$ & $46(34.8 \%)$ & $N S$ \\
\hline Loop diuretics & $120(43.2 \%)$ & $74(56.1 \%)$ & $<0.05$ \\
\hline $\mathrm{K}^{+}$-sparing diuretics & $42(15.1 \%)$ & $23(17.4 \%)$ & NS \\
\hline Digitalis glycosides & $49(17.6 \%)$ & $38(28.8 \%)$ & $<0.01$ \\
\hline Aspirin & $87(31.3 \%)$ & $32(24.2 \%)$ & NS \\
\hline Ticlopidine & $30(10.8 \%)$ & $8(6.1 \%)$ & NS \\
\hline Oral anticoagulants & $98(35.3 \%)$ & $32(24.2 \%)$ & $<0.05$ \\
\hline Nitrates & $59(21.2 \%)$ & $26(19.7 \%)$ & NS \\
\hline Statins & $55(19.8 \%)$ & $20(15.2 \%)$ & NS \\
\hline Electrocardiographic data & & & \\
\hline LV hypertrophy & $50(18.0 \%)$ & $27(20.5 \%)$ & $N S$ \\
\hline Left bundle branch block & $17(6.1 \%)$ & $6(4.5 \%)$ & $N S$ \\
\hline Right bundle branch block & $18(6.5 \%)$ & $7(5.3 \%)$ & $N S$ \\
\hline Pathologic $Q$-wave & $60(21.6 \%)$ & $11(8.3 \%)$ & $<0.01$ \\
\hline Echocardiographic data & & & \\
\hline Ejection fraction (\%) & $39.4 \pm 8.8$ & $41.2 \pm 8.1$ & $<0.05$ \\
\hline Fractional shortening (\%) & $19.8 \pm 5.1$ & $20.5 \pm 4.3$ & $N S$ \\
\hline $\operatorname{LVDd}(\mathrm{mm})$ & $56.4 \pm 9$ & $54.3 \pm 8.2$ & $<0.05$ \\
\hline $\operatorname{LVDs}(\mathrm{mm})$ & $45.7 \pm 7.6$ & $43.3 \pm 7.6$ & $<0.01$ \\
\hline $\operatorname{IVST}(\mathrm{mm})$ & $10.5 \pm 3.0$ & $9.6 \pm 2.7$ & $<0.01$ \\
\hline$P W T(\mathrm{~mm})$ & $10.4 \pm 2.4$ & $9.8 \pm 2.1$ & $<0.05$ \\
\hline LV mass index $\left(\mathrm{g} / \mathrm{m}^{2}\right)$ & $149.5 \pm 52.0$ & $145.9 \pm 46.9$ & NS \\
\hline
\end{tabular}




Left atrial diameter (mm)
Tricuspid valve regurgitation
Mitral valve regurgitation
Aortic valve regurgitation
Functional and prognostic indices
New York Hear Association functional class
Class I
Class II-III
Class IV
Charlson comorbidity index
0 point
1 point
2 points
3 points
$\geq 4$ points

$41.9 \pm 8.8$
$45(16.2 \%)$
$28(10.1 \%)$
$12(4.3 \%)$

$74(26.5 \%)$
$193(69.5 \%)$
$11(4.0 \%)$
$57(20.5 \%)$
$76(27.3 \%)$
$69(24.8 \%)$
$43(15.5 \%)$
$33(11.9 \%)$

$43.3 \pm 9.7$

$27(20.5 \%)$

$20(15.2 \%)$

$7(5.3 \%)$

$N S$

NS

NS

NS

$<0.05$

$25(19.0 \%)$

$0(0 \%)$
07

$41(31.1 \%)$

$34(25.8 \%)$

$32(24.2 \%)$

$11(8.3 \%)$

$14(10.6 \%)$

*Survived until January $1^{\text {st }} 2003$.

Echocardiography data shown are those obtained on the initial diagnosis of SD. LV mass $(g)$ was calculated according to the method recommended by the American Society of Echocardiography $\left[L V\right.$ mass $\left.=0.8 \times\left(1.04 \times[L V D d+I V S T+P W T]^{3}-L V D d^{3}\right)+0.6\right]$ then was indexed to body surface area $\left(\mathrm{m}^{2}\right)$.

CHF, congestive heart failure; CRI, chronic renal impairment; CRF, chronic renal failure; ACEIs, angiotensin-converting enzyme inhibitors; LV, left vantricular; LVDd, left vantricular diastolic dimension; LVDs, left vantricular systolic dimension; IVST, interventricular septal thickness; PWT, posterior wall thickness.

Values are mean $\pm S D$ or number of subjects (\%).

Table 3 Variables Independently Associated With Mortality*

\begin{tabular}{lcccr}
\hline \hline & B-coefficient & OR & $95 \%$ CI of OR & p value \\
\hline Body mass index $\left(\mathrm{kg} / \mathrm{m}^{2}\right)$ & -0.168 & 0.845 & $0.796-0.898$ & $<0.001$ \\
Age at diagnosis & 0.033 & 1.034 & $1.012-1.055$ & 0.002 \\
LVDd $\times$ LVDs interaction $\left(\mathrm{cm}^{2}\right)$ & 0.039 & 1.040 & $1.020-1.060$ & $<0.001$ \\
CHF history & 0.525 & 1.690 & $1.218-2.346$ & 0.002 \\
Malignancy & 0.788 & 2.199 & $1.492-3.242$ & $<0.001$ \\
Stroke & 0.798 & 2.221 & $1.591-3.101$ & $<0.001$ \\
Chronic kidney disease & 0.921 & 2.511 & $1.655-3.808$ & $<0.001$ \\
\hline
\end{tabular}

*Using stepwise Cox proportionate hazard regression model, with the corresponding ORs.

OR, odds ratio; CI, confidence interval. Other abbreviations see in Table 2.

\section{Predictors of Mortality}

A Cox proportionate hazard regression model was constructed for identifying the prognostic predictors of death. Simple univariate analysis was done first for each of the input variables against positive death outcome, and all the variables (except for gender) associated with it at $p \leq 0.1$ were finally entered in a multivariate backward stepwise Cox regression model. Owing to the high correlation between LV systolic dimension (LVDs) and LV diastolic dimension (LVDd), as well as LVEF and fraction shortening (FS), these 2 pairs of variables were entered into the model as interaction terms (ie, LVDd $\times$ LVDs in $\mathrm{cm}^{2}$ and LVEF $\times$ FS) to avoid collinearity. Thus, the following variables were included in the final model: age at diagnosis, gender, body mass index (BMI), presence of dyslipidemia, history of CHF, chronic kidney disease (CKD) (serum creatinine $\geq 2 \mathrm{mg} / \mathrm{dl}$ or regular hemodialysis), stroke, malignancy, treatment with angiotensin-converting enzyme inhibitors or diuretics, and the interactions of LVDd/LVDs and EF/FS. The model was proven to be highly significant (p-value for the omnibus tests of model coefficients $<0.001$ ). Eventually, age at primary diagnosis, LVDd $\times$ LVDs interaction, history of CHF, presence of stroke, CKD, and malignancy were found to be significantly and independently associated with death, and BMI was an independent inverse correlate of that event (Table 3).

\section{Discussion}

Evaluating the burden of cardiovascular disease on society has become an urgent requirement because disease profiles have changed rapidly in accordance with aging of the general population. The Niigata-Sado Heart Failure Study was conducted to provide such information. The demo-geographic characteristics of the study area (ie, an island) facilitated follow-up of the study cohort and improved subject inclusiveness and the sequentiality of enrollment, giving the advantage of reducing bias in patient selection. From this study, we have the following new observations: (1) the number of outpatients with SD amount to $2-3 \%$ of the population; (2) there is a gender difference in the characteristics of patients with SD; (3) patients with SD have several comorbidities that affect prognosis; and (4) the prognosis of patients with SD, even with favorable LVEF (41-50\%), is worse than that of the general population.

The aim of this study was to count the number of outpatients with SD in 2003 and clarify their characteristics. Before doing so, we verified whether recruitment period was appropriate (Fig 2), and SD patients were collected without regional difference on the island (Fig 5). The dates when the patients were diagnosed to have SD varied; for example, when considering 2 patients who were diagnosed in 2002 and in 1999, the recruitment from 2002 to 2003 could identify the former but not the latter, whereas recruitment from 1999 to 2003 could identify both. Because there 
was a directly proportional relationship between the number of identified patients and the length of the recruitment period; high inclusiveness was expected by setting the starting point of the period as early as possible (even more than 5 years) as shown in Fig 2. However, some SD patients died before 2003, and there was also an incremental relationship between the number of SD patients who died before 2003 and the length of the recruitment period. Therefore, the net increase in SD survivors by 2003 was suppressed by extending the recruitment period (Fig 2). Thus, we believe that the 5-year recruitment period (from 1998 to 2002) was efficient and appropriate for counting SD survivors in 2003.

The proportion of SD patients in the general population sector aged $45-84$ years was $1.5 \%$ for males and $0.6 \%$ for females (Fig 4). This proportion increased after the age of 65 years in males and 70 years in females, reaching $3.3 \%$ in males and $1.7 \%$ in females in their $80 \mathrm{~s}$. These estimates were only based on the number of patients who were confirmed to have SD, and those in the general population with latent cardiac dysfunction who did not undergo Echo examinations were not included. Therefore, the estimates presented here are virtually lower than the true counts, and more residents may have untreated SD. These estimates differ considerably from the prevalence rates reported by population-based studies in the United States and Europe (6.9-10.2\% for males and 3.4-3.8\% for females; $11.2 \%$ for males and $8.3 \%$ for females in their $80 \mathrm{~s})^{2,3}$ In fact, detailed comparison would be impossible for many reasons (eg, geo-demographic, ethnic and methodological differences); however, the lower incidence of MI in the Japanese population compared with those of Western countries may be the reason for the lower prevalence of SD in Japan! ${ }^{15,16}$

Our results highlighted the average characteristics of patients who were often clinically observed with the least possible selection bias, unlike the data derived from largescale clinical studies conducted on subjects who have passed multiple exclusion criteria. Tsutsui et al $^{17}$ and Shiba et $\mathrm{a}^{18}$ reported the clinical characteristics of Japanese patients with CHF in Fukuoka and Tohoku, respectively. The prevalence of hypertension, IHD, and atrial fibrillation (AF) was $39-41 \%, 25-44 \%$, and $31-41 \%$, respectively. Compared with their cohorts in which most patients had a history of hospital admission for CHF, the majority (71\%) of our SD cohort did not have such a history. However, the prevalences of hypertension (40\%), IHD (34\%), and AF $(31 \%)$ were consistent with these previous reports. Senni et $\mathrm{al}^{19}$ reported the clinical characteristics of white patients who were admitted with new-onset CHF in Olmsted County. They categorized 137 patients into $\mathrm{CHF}$ with reduced LVEF $(<50 \%)$ group and that with preserved LVEF $(\geq 50 \%)$. Seventy-eight patients (57\%) showed reduced LVEF, males were dominant $(59 \%)$, and the average age was 74.2 years. The prevalences of hypertension, IHD, AF and DCM were $50 \%, 53 \%, 24 \%$, and $3 \%$ respectively. Hypertension and IHD prevailed more in the white cohorts than in ours, whereas AF and DCM were more prevalent in our cohort.

Comorbidity was a significant health problem for the patients with SD in this study. Half of them had a CCI score $\geq 2$ (Table 2), and were thus considered as a poor prognosis group ${ }^{20,21}$ Compared with the reports from North America, ${ }^{20,21}$ our cohort had a higher CCI score; for example, the proportion of patients (or residents) with CCI score $\geq 2$ in the Duke databank of IHD (1,471 patients, 61 years old on average $)^{20}$ the population-based cohort of Olmsted county (2,037 people, average age of 61 years $)^{22}$ and in the HF registry of Ontario $(38,702$ patients, $85 \%$ of the patients were over 65 years old $)^{21}$ was only $19 \%, 32 \%$, and $29 \%$, respectively. The reasons for the higher CCI score in our cohort are not clear, but we hypothesize higher age, the hospital-based study design, and the meticulous archiving of patients data, which maximized the diagnosis of associated comorbidities.

Additionally, some of the comorbidities (CHF, stroke, CKD, and malignancy), which were also scored by CCI, were shown to be independently and significantly associated with death in our cohort. In general, the high prevalence of poor prognostic comorbidities worsens the survival of the cohort. Therefore, the survival of these patients was poorer than that of the general population, in either those with relatively favorable systolic function (LVEF 41-50\%) or those with significantly impaired LVEF $(\leq 40 \%)$ (Fig 6).

\section{Study Limitations}

First, because this study was hospital-based and not population-based, it might have missed the latent SD patients in the community. In Sado City, $43 \%$ of residents over 45 years annually undergo medical check-up for early detection of disease, which is supported by the local government. However, we cannot exactly define the proportion of residents who proceeded to Echo examinations out of the total number examined clinically. Accordingly, estimation of the precise prevalence of SD in the general population was not possible, so instead we have shown the proportion of Echo-documented SD. Also, the literature has indicated a gender difference regarding consultation, examination, and treatment of cardiac patients, 23 which our study could not avoid. Moreover, the limitation of the study area to Sado Island may restrict the generalization of our results to other areas in Japan. In addition, half of the patients, especially asymptomatic ones with mild SD, did not undergo diagnostic coronary angiography. In this respect, it is known whether noninvasive diagnostic processes have limitations in classifying disease etiology precisely.

\section{Conclusions}

We carried out the first complete enumeration survey of SD in a Japanese community. The Sado Heart Failure Study is a highly inclusive hospital-based study that may be able to characterize patients with SD in the community. The proportion of patients with SD in the general population increases progressively with age. These patients often have multiple comorbidities, making the outcome of even mild $\mathrm{SD}$ a poor one. The gender difference in disease characteristics and living conditions of patients should be taken into consideration when establishing preventive strategies for HF in Japanese communities.

\section{Acknowledgments}

This study was performed in collaboration with Dr Akira Hattori, Director, and Dr Takeshi Momotsu, Vice-Director of Sado General Hospital, Drs Kenjiro Kikuchi, Takashi Ishikawa, Yoshio Kitami, and Keiko Saito of the Physiologic Laboratory, and the physicians of the Sado Medical Society.

It was supported by a Grant-in-Aid for Basic Research on Science and Technology $(C)$ : "Comprehensive comparative evaluation of risk factors for hospitalization and death of patients with chronic heart failure in urban and rural regions" (17606003). 


\section{References}

1. Braunwald E. Shattuck lecture: Cardiovascular medicine at the turn of the millennium: Triumphs, concerns, and opportunities. $N$ Engl $J$ Med 1997; 337: 1360-1369.

2. Redfield MM, Jacobsen SJ, Burnett JC Jr, Mahoney DW, Bailey KR, Rodeheffer RJ. Burden of systolic and diastolic ventricular dysfunction in the community: Appreciating the scope of the heart failure epidemic. JAMA 2003; 289: 194-202.

3. Davies M, Hobbs F, Davis R, Kenkre J, Roalfe AK, Hare R, et al. Prevalence of left-ventricular systolic dysfunction and heart failure in the Echocardiographic Heart of England Screening study: A population based study. Lancet 2001; 358: 439-444.

4. Mosterd A, Hoes AW, de Bruyne MC, Deckers JW, Linker DT, Hofman A, et al. Prevalence of heart failure and left ventricular dysfunction in the general population: The Rotterdam study. Eur Heart $J$ 1999; 20: 447-455.

5. Hunt SA, Abraham WT, Chin MH, Feldman AM, Francis GS, Ganiats TG, et al; American College of Cardiology; American Heart Association Task Force on Practice Guidelines; American College of Chest Physicians; International Society for Heart and Lung Transplantation; Heart Rhythm Society. ACC/AHA 2005 Guideline Update for the Diagnosis and Management of Chronic Heart Failure in the Adult: A report of the American College of Cardiology/American Heart Association Task Force on Practice Guidelines (Writing Committee to Update the 2001 Guidelines for the Evaluation and Management of Heart Failure): Developed in collaboration with the American College of Chest Physicians and the International Society for Heart and Lung Transplantation: Endorsed by the Heart Rhythm Society. Circulation 2005; 112: e154-e235.

6. Swedberg K, Cleland J, Dargie H, Drexler H, Follath F, Komajda M, et al; Task Force for the Diagnosis and Treatment of Chronic Heart Failure of the European Society of Cardiology. Guidelines for the diagnosis and treatment of chronic heart failure: Executive summary (update 2005): The Task Force for the Diagnosis and Treatment of Chronic Heart Failure of the European Society of Cardiology. Eur Heart J 2005; 26: 1115-1140.

7. Department of Economic and Social Affairs, Population Division of United Nations. Percentage distribution of the population in selected age groups, by country, 2005 and 2050. In: World population prospects: The 2004 revision (highlights). New York: United Nations; 2005; 53-57.

8. McKee PA, Castelli WP, McNamara PM, Kannel WB. The natural history of congestive heart failure: The Framingham study. $N$ Engl J Med 1971; 285: $1441-1446$.

9. Division of Statistics, Department of Policy and Planning, Niigata, Japan. Population estimates of Niigata prefecture, 2003. Niigata: The Department, 2003 (in Japanese).
10. Saunders BD, Trapp RG. Basic and clinical biostatistics, 2nd edn. Norwalk, CN: Appleton \& Lange, 1994.

11. Amitage P, Berry G. Statistical methods in medical research, 3rd edn. Oxford, UK: Blackwell Science, 2001.

12. Charlson ME, Pompei P, Ales KL, MacKenzie CR. A new method of classifying prognositic comorbidity in longitudinal studies: Development and validation. J Chronic Dis 1987; 40: 373-383.

13. Charlson M, Szatrowski TP, Peterson J, Gold J. Validation of a combined comorbidity index. J Clin Epidemiol 1994; 47: 1245-1251.

14. Chin MH, Goldman L. Correlates of early hospital readmission or death in patients with congestive heart failure. Am J Cardiol 1997; 79: $1640-1644$.

15. Menotti A, Puddu PE, Lanti M, Kromhout D, Blackburn H, Nissinen A. Twenty-five-year coronary mortality trends in the seven countries study using the accelerated failure time model. Eur J Epidemiol 2003; 18: $113-122$.

16. Verschuren WM, Jacobs DR, Bloemberg BP, Kromhout D, Menotti A, Aravanis C, et al. Serum total cholesterol and long-term coronary heart disease mortality in different cultures: Twenty-five-year followup of the seven countries study. JAMA 1995; 274: 131-136.

17. Tsutsui H, Tsuchihashi M, Takeshita A. Mortality and readmission of hospitalized patients with congestive heart failure and preserved versus depressed systolic function. Am J Cardiol 2001; 88: 530533.

18. Shiba N, Watanabe J, Shinozaki T, Koseki Y, Sakuma M, Kagaya Y, et al; CHART Investigators. Analysis of chronic heart failure registry in the Tohoku District: Third year follow up. Circ J 2004; 68: $427-$ 434.

19. Senni M, Tribouilloy CM, Rodeheffer RJ, Jacobsen SJ, Evans JM, Bailey KR, et al. Congestive heart failure in the community: A study of all incident cases in Olmsted County, Minnesota, in 1991. Circulation 1998; 98: 2282-2289.

20. Sachdev M, Sun JL, Tsiatis AA, Nelson CL, Mark DB, Jollis JG. The prognostic importance of comorbidity for mortality in patients with stable coronary artery disease. J Am Coll Cardiol 2004; 43: $576-582$.

21. Jong P, Vowinckel E, Liu PP, Gong Y, Tu JV. Prognosis and determinants of survival in patients newly hospitalized for heart failure: A population-based study. Arch Intern Med 2002; 162: 1689-1694.

22. Okura Y, Urban LH, Mahoney DW, Jacobsen SJ, Rodeheffer RJ. Agreement between self-report questionnaires and medical record data was substantial for diabetes, hypertension, myocardial infarction and stroke but not for heart failure. J Clin Epidemiol 2004; 57: 1096-1103.

23. Gan SC, Beaver SK, Houck PM, MacLehose RF, Lawson HW, Chan L. Treatment of acute myocardial infarction and 30-day mortality among women and men. N Engl J Med 2000; 343: 8-15. 